\title{
Clear cap-assisted luminal stenting may improve technical success in gastroduodenal and colonic obstruction
}

\section{다 (1) $\odot$}

\section{Authors}

Brian M. Fung ${ }^{1}$, Formosa C. Chen², James H. Tabibian ${ }^{3,4}$

Institutions

1 Department of Medicine, Olive View-UCLA Medical Center, Sylmar, California, United States

2 Department of Surgery, Olive View-UCLA Medical Center, Sylmar, California, United States

3 Division of Gastroenterology, Department of Medicine, Olive View-UCLA Medical Center, Sylmar, California, United States

4 David Geffen School of Medicine at UCLA, Los Angeles, California, United States

submitted 19.2.2020

accepted after revision 11.5 .2020

\author{
Bibliography \\ Endoscopy International Open 2020; 08: E1429-E1434 \\ DOI 10.1055/a-1229-4000 \\ ISSN 2364-3722 \\ (c) 2020. The Author(s).
}

This is an open access article published by Thieme under the terms of the Creative Commons Attribution-NonDerivative-NonCommercial License, permitting copying and reproduction so long as the original work is given appropriate credit. Contents may not be used for commecial purposes, or adapted, remixed, transformed or built upon. (https://creativecommons.org/licenses/by-nc-nd/4.0/)

Corresponding author

James H. Tabibian, MD, PhD, FACP, Director of Endoscopy, Housestaff Research Director, Olive View-UCLA Medical Center, 14445 Olive View Dr., 2B-182, Sylmar, CA 91342, United States

Fax: +1-747-210-4573

jtabibian@dhs.lacounty.gov

\section{ABSTRACT}

Background Luminal stenting is safe, effective, and at times the preferred method for relieving gastrointestinal obstruction. However, stent placement is not technically feasible when lesions cannot be traversed with a guidewire, resulting in the need for more invasive methods of palliation and urgent/emergent surgical intervention. In this series, we report our experience with cap-assisted stenting to improve lumen visualization in obstructed segments of the gastrointestinal tract and salvage cases that may have otherwise resulted in technical failure.

Methods A clear cap (i. e. distal attachment) was affixed to the scope tip to facilitate visualization and stenting in two cases of gastroduodenal obstruction and three cases of colonic obstruction. Indications for stent placement included malignant obstruction, diverticulitis-associated obstruction, and Crohn's disease-associated stricture.

Results In this report, we demonstrate that use of a clear cap facilitated endoscopic stenting in challenging cases of malignant and benign gastroduodenal and colonic obstruction.

Conclusions A clear cap for endoscopic stenting can be used in cases in which standard techniques are unsuccessful or those anticipated to be technically difficult at the outset. Cases with tortuous anatomy or particularly tight, friable, or exophytic obstructive lesions may benefit most from this novel technical modification.

\section{Introduction}

Self-expanding metallic stents (SEMSs) are commonly used to palliate luminal obstruction in the gastrointestinal tract. However, SEMS placement is not always technically feasible, in some instances due to difficulty visualizing the luminal orifice and subsequently passing the guidewire through the site of obstruction [1,2]. In recent years, cap-assisted endoscopy (the use of a distal attachment over the endoscope tip) has been found to facilitate various procedures, such as visualization of obscured lesions during colonoscopy (e.g. polyps behind colo-

nic haustral folds), resection of benign or mucosal-based malignant lesions, and cannulation during enteroscope-assisted endoscopic retrograde cholangiopancreatography (ERCP) [310].

We recently found that use of a clear cap facilitated endoscopic SEMS placement in a challenging case of malignant colonic obstruction that otherwise would have been a technical failure requiring emergent diverting colostomy. Based on this initial experience, we sought to assess the potential utility of clear cap use in increasing technical success in other challenging cases of luminal stenting. 

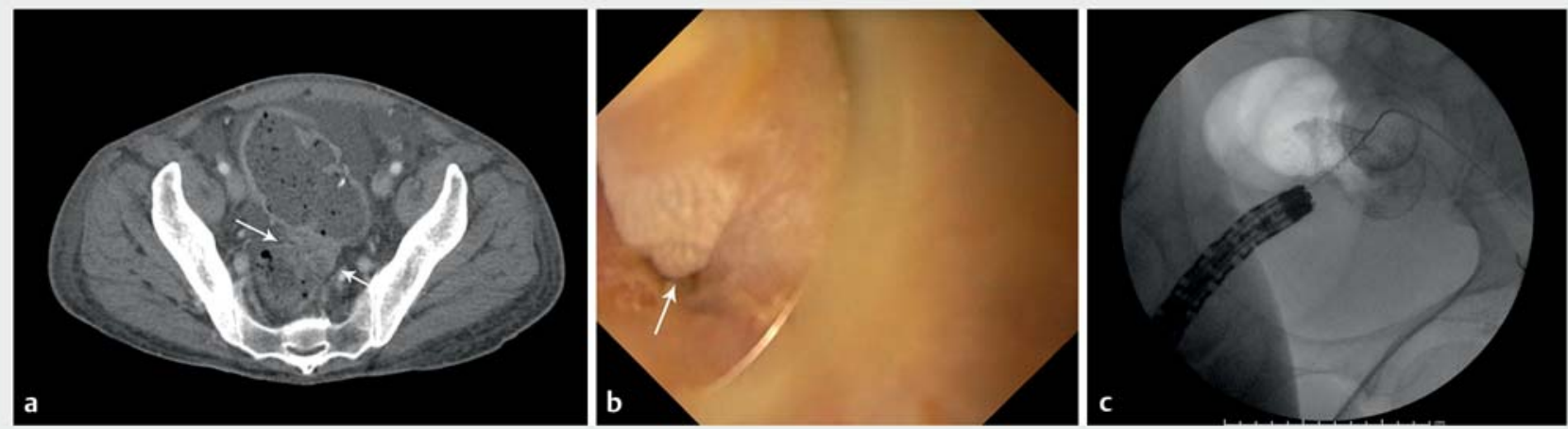

- Fig. 1 A 46-year-old male with malignant sigmoid obstruction. a Computed tomography (CT) of the abdomen and pelvis shows a mass (arrow) and transition point in the sigmoid colon with associated proximal colonic dilation. b Visualization of a diminutive stricture orifice (arrow), though initially unsuccessful by conventional techniques, was made possible by use of a clear cap.c Stent deployment through a tortuous, tight stricture under fluoroscopic guidance.

\section{Methods}

All procedures were performed at Olive View-UCLA Medical Center, a 377-bed safety net hospital and regional referral center serving the northern catchment area of the Los Angeles County Department of Health Services, the second largest municipal healthcare system in the United States. Many of the luminal stenting procedures performed at our institution are technical failures from outside facilities that are referred for a higher level of care. The average technical success rate for endoscopic luminal stenting at our institution has historically been $>95 \%$.

Between February 2018 and April 2018, 16 upper and lower endoscopies with luminal (gastroduodenal or colonic) stent placement were performed at our institution. In five of these 16 cases (31\%), a clear cap (3-mm length distal attachment, Olympus America, Center Valley, Pennsylvania, United States) was attached to the scope tip to salvage what would have likely otherwise been a technical failure. The remaining 11 cases (69\%) were successfully performed with conventional techniques without needing a cap. All procedures were performed with Olympus (EVIS EXERA, Olympus America) endoscopes, as detailed below.

\section{Case reports}

\section{Patient 1: Malignant sigmoid obstruction}

A 46-year-old male with metastatic sigmoid adenocarcinoma on palliative chemotherapy presented with 1 week of constipation, abdominal distension, and pain. Computed tomography (CT) of the abdomen and pelvis showed colonic obstruction with a transition point in the sigmoid colon and associated proximal colonic dilation ( $\boldsymbol{F}$ Fig. 1a). The patient was referred for emergent colonic stenting. A gastrointestinal F-1TQ160 therapeutic gastroscope was advanced to the distal sigmoid, where a firm, malignant-appearing lesion was encountered completely occupying the lumen ( $\triangleright$ Fig. 1b). Multiple attempts to pass a guidewire were unsuccessful. The scope was with- drawn, and a diagnostic upper endoscope (gastrointestinal FH190) was used to provide more dexterity and flexibility in light of the fixed, angulated sigmoid. However, the stricture lumen still could not be visualized nor could a guidewire be passed through it. The scope was withdrawn, and a clear cap was attached to improve visualization. With cap facilitation, a pinpoint orifice was visualized, and a wire was successfully passed through the site of obstruction, permitting placement of a $25 \mathrm{~mm} \times 90 \mathrm{~mm}$ WallFlex (Boston Scientific, Marlborough, Massachusetts, United States) colonic SEMS ( $>$ Fig.1c). There was satisfactory decompression of the colon, and the patient was subsequently discharged from the hospital on a low-residue diet.

\section{Patient 2: Malignant gastric outlet obstruction}

A 54-year-old male with metastatic gastric adenocarcinoma presented for progressive abdominal pain and oral intolerance. CT revealed irregular, circumferential wall thickening of the gastric antrum. During upper endoscopy, a bulky $(6 \mathrm{~cm})$, friable mass was found to completely occupy the antrum ( $\mathbf{F i g . 2 a ) ; ~}$ no lumen was identifiable. Despite multiple attempts under endoscopic and fluoroscopic guidance with the assistance of pressure injection of contrast, wire access across the stricture could not be attained ( $\mathbf{F i g . 2 b}$ ). The scope was removed, a clear cap attached, and re-advanced. With cap-assistance, a pinpoint lumen was found ( $\mathbf{F i g . 2 c}$ ), traversed with a guidewire, and a $22 \mathrm{~mm} \times 90 \mathrm{~mm}$ WallFlex duodenal SEMS successfully placed ( $\triangleright$ Fig. $\mathbf{2 d}$ d $\triangleright$ Fig. 2e, $\triangleright$ Fig. 2f). Contrast was shown flowing through the stent ( $\mathbf{F i g . 2} \mathbf{g}$ ). The patient was discharged to home hospice the following day; he passed away 6 weeks later.

\section{Patient 3: Crohn's disease-associated colonic obstruction}

A 53-year-old male with longstanding Crohn's disease (reportedly Crohn's colitis) presented with 1 month of progressive left lower quadrant abdominal pain, decreased appetite, and weight loss. CT revealed a mass-like lesion in the descending 

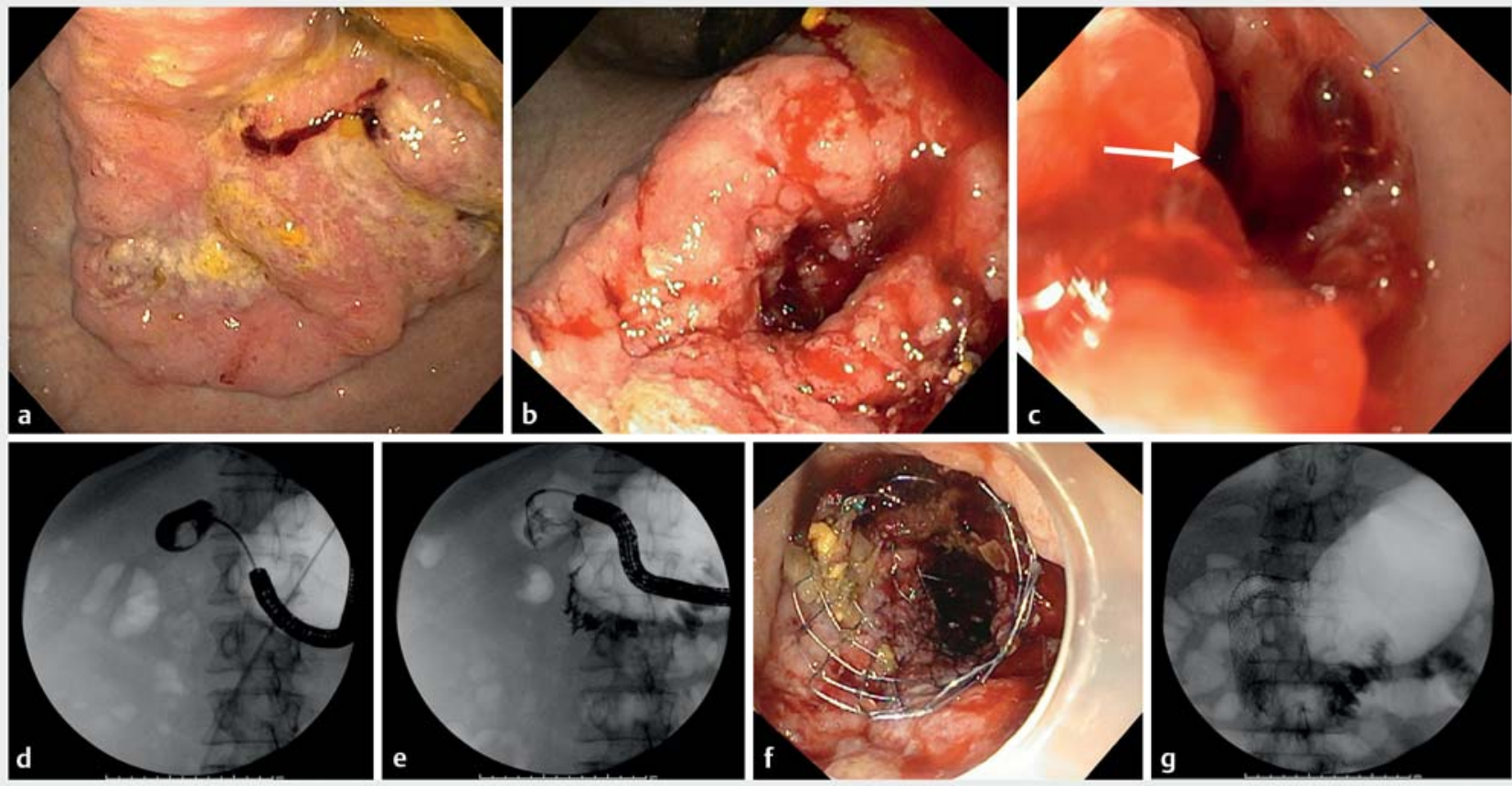

Fig.2 A 54-year-old male with metastatic gastric adenocarcinoma. a A 6-cm flat, friable mass is seen in the gastric antrum during upper endoscopy. $\mathbf{b}$ Multiple attempts to find a lumen by conventional techniques were unsuccessful, leading to bleeding of friable tumor tissue. c Visualization of the stricture lumen (arrow) was improved via cap assistance (blue line). d Fluoroscopy revealed lack of contrast flow through the lesion. e With pressure (i. e. occlusion) injection of contrast, a diminutive and tortuous lumen could be seen. $\mathbf{f}$ View of the proximal end of the duodenal stent. g Contrast can be seen flowing through the deployed duodenal stent.
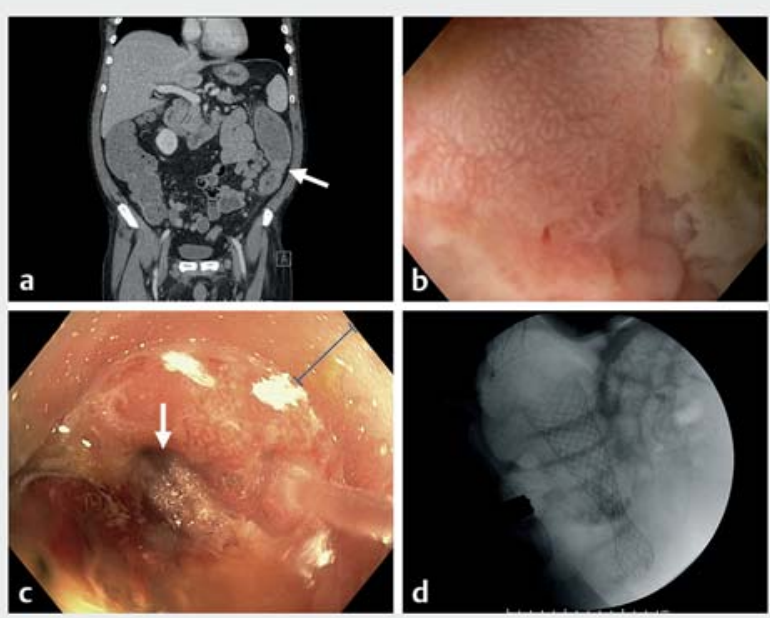

- Fig. 3 A 53-year old male with Crohn's colitis and a colonic stricture. a CT demonstrating a region of focal colonic narrowing in the distal descending/sigmoid colon (arrow, coronal view). b An edematous, ulcerated, circumferentially obstructing lesion is seen during colonoscopy, without a clear luminal orifice. c Extensive irrigation and use of a clear cap (blue line) facilitated visualization of the stricture lumen (arrow). d Fluoroscopy confirmed successful stent deployment across the stricture. colon with marked proximal distention ( $\triangleright$ Fig. 3a). Based on the patient's presentation, long history of Crohn's disease, absence of biochemical evidence of active inflammation, and the CT findings, there was strong suspicion for a malignant neoplasm. A multidisciplinary decision was made to place a colonic stent to decompress the acute obstruction and facilitate a singlestage, non-emergent surgical resection, recognizing that other colonoscopic maneuvers such as balloon dilation of a possibly malignant lesion would ostensibly be ineffective or higher risk (bleeding, perforation). During colonoscopy, an ulcerated obstructing lesion ( $\vee$ Fig. $\mathbf{3 b}$ ), corresponding to the mass seen on $\mathrm{CT}$, was encountered and biopsied. Identification of the lumen was hindered by fecal debris and adjacent diverticula. The scope was thus removed, and a clear cap was attached to better examine the stricture ( $\vee$ Fig. $3 c$ ). With cap assistance, the stricture lumen was identified, and a guidewire was successfully passed through it. A $25 \mathrm{~mm} \times 90 \mathrm{~mm}$ WallFlex colonic SEMS was then deployed ( $\vee$ Fig.3 d) with immediate symptomatic improvement. Two days post-stenting, the patient reported increased pelvic discomfort. Repeat CT imaging revealed significant decompression of the colon, improved appearance of the strictured segment, and SEMS migration into the rectum. The SEMS was colonoscopically retrieved, and the strictured segment was re-biopsied. Pathology revealed a benign stricture, which was confirmed on a subsequent, fully-prepped colonoscopy with repeat biopsies. On last follow-up (10 months thereafter), the patient has remained without obstructive symptoms 

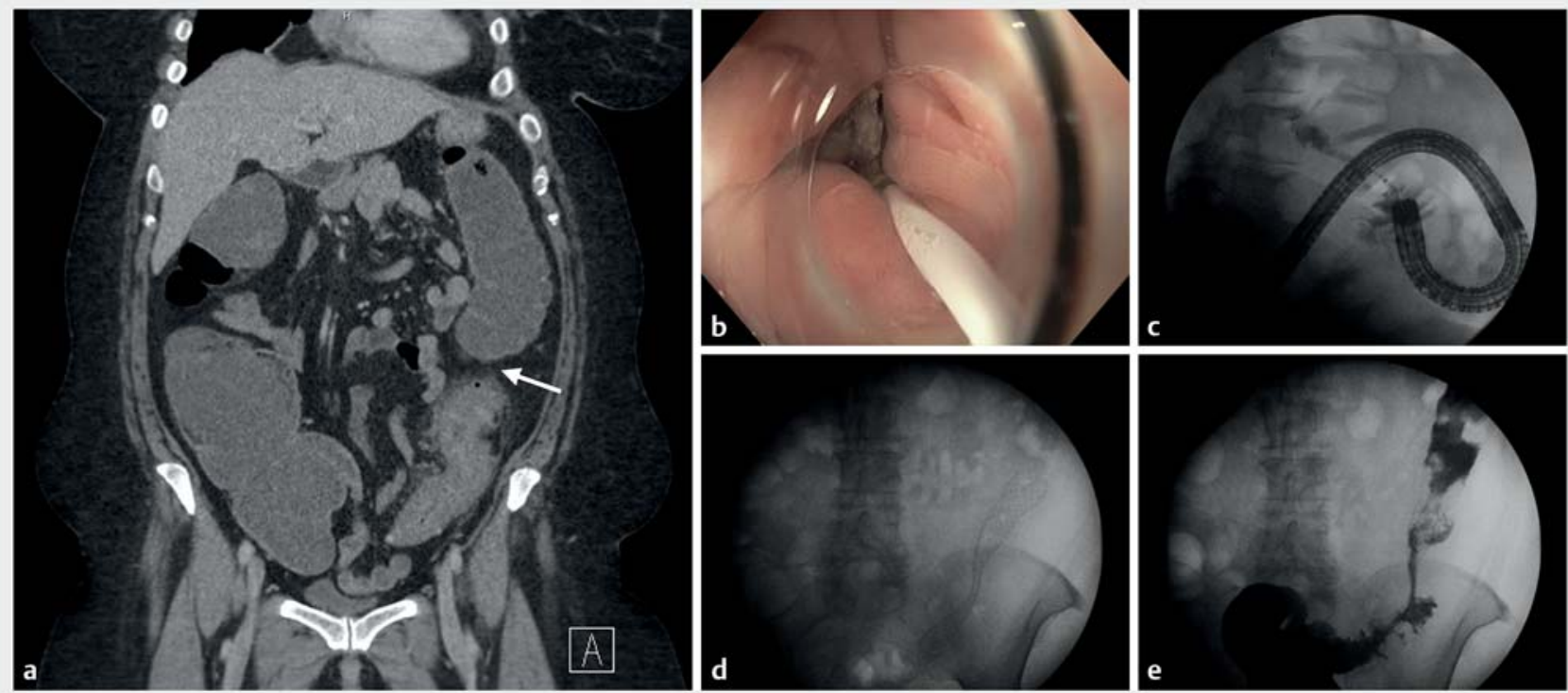

- Fig. 4 A 58-year-old female with presumed diverticulitis-associated colonic obstruction. a CT prior to stenting shows a distal descending/ proximal sigmoid colon stricture (arrow) and associated diverticula and pericolonic inflammation. $\mathbf{b}$ Improved visualization of the stricture orifice was achieved with cap assistance. $\mathbf{c}$ Fluoroscopy showing extent of angulation and tortuosity of the stricture. $\mathbf{d}$ Post-stenting abdominal $\mathrm{X}$-ray demonstrating the length and tortuosity of the stricture as well as the improved bowel gas pattern post-stenting, and e post-stenting gastrograffin enema confirming that the colonic stent is patent.

and has been restarted on pharmacotherapy for Crohn's disease.

\section{Patient 4: Diverticulitis-associated colonic obstruction}

A 58-year-old female with recurrent acute diverticulitis presented with 4 days of abdominal pain, distension, and obstipation. CT revealed sigmoid inflammation suggestive of acuteon-chronic diverticulitis and colonic obstruction with cecal diameter of approximately $8 \mathrm{~cm}$ ( $\mathbf{F i g . 4 a )}$. To relieve the obstruction and increase the likelihood of a one-stage, stomafree segmental colectomy, the surgical team requested placement of a colonic stent. Upon colonoscopy, diverticulosis was seen within a tortuous sigmoid colon, and at approximately $35 \mathrm{~cm}$ from the anal verge, the lumen became obscured. There was circumferential mucosal erythema, congestion, and edema, consistent with diverticulitis. Given the friability, sharp angulation, and dense diverticulosis in the region, identification of the true lumen was difficult. Outfitting the colonoscope with a clear cap, a lumen was successfully identified, but the obstructed segment still could not be traversed. A diagnostic gastroscope with a clear cap was thus used to visualize the orifice of the stricture lumen and permitted guidewire traversal through it ( $\vee$ Fig.4b). The obstructed segment measured approximately $6 \mathrm{~cm}$ in length fluoroscopically ( $\mathbf{F i g . 4 c}$, - Fig. 4d), and a $25 \mathrm{~mm} \times 120 \mathrm{~mm}$ WallFlex colonic SEMS was successfully deployed across it ( $\boldsymbol{\sim}$ Fig. $4 \mathbf{4}$ ). A single-stage sigmoid colectomy was performed 2 weeks later, and on 1-year follow up, the patient was doing well.
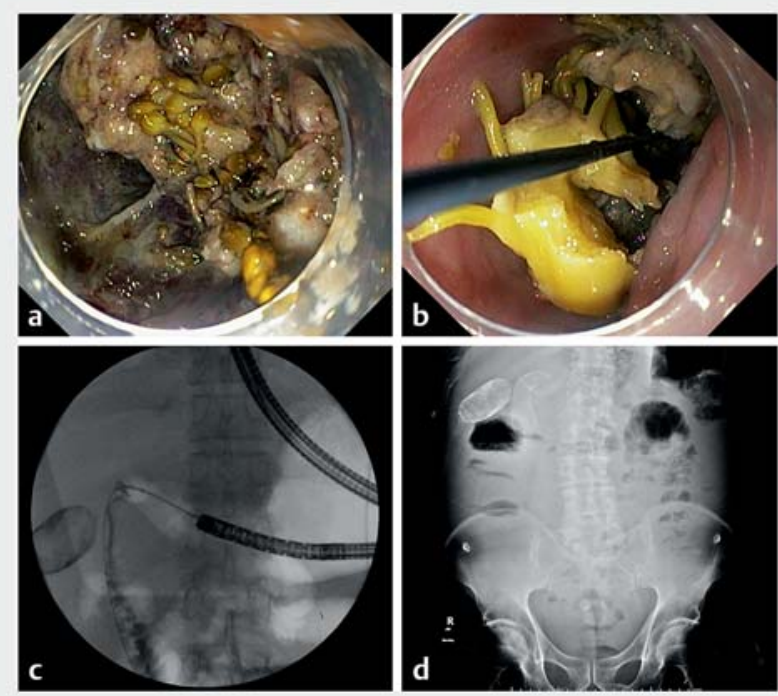

Fig.5 A 62-year-old male with malignant gastric outlet obstruction. a A large fungating, ulcerated, mass is seen in the gastric body and antrum during endoscopy (performed under general anesthesia for airway protection), as well as a large volume of food debris. b With cap assistance, a luminal orifice was identified and a guidewire successfully advanced through the lumen of the obstructed segment. c Contrast injection under fluoroscopy shows the length and severity of the stricture. $\mathbf{d}$ Post-stenting $\mathrm{x}$-ray demonstrates successful stent placement. 
- Table 1 Summary of cases of endoscopic cap-assisted luminal stenting.

\begin{tabular}{|c|c|c|c|c|c|c|c|}
\hline Case & Age & Sex & Indication & Underlying Diagnosis & Procedure & Result $^{1}$ & $\begin{array}{l}\text { Adverse } \\
\text { Events }^{2}\end{array}$ \\
\hline 1 & 46 & M & Sigmoid obstruction & $\begin{array}{l}\text { Metastatic colorectal } \\
\text { adenocarcinoma }\end{array}$ & Colonic stent placement & Success & None \\
\hline 2 & 54 & M & Gastric outlet obstruction & $\begin{array}{l}\text { Metastatic gastric adeno- } \\
\text { carcinoma }\end{array}$ & Duodenal stent placement & Success & None \\
\hline 3 & 53 & M & $\begin{array}{l}\text { Descending colon } \\
\text { obstruction }\end{array}$ & Crohn's colitis stricture & Colonic stent placement & Success & None \\
\hline 4 & 58 & $\mathrm{~F}$ & Sigmoid obstruction & $\begin{array}{l}\text { Diverticulitis-associated } \\
\text { stricture }\end{array}$ & Colonic stent placement & Success & None \\
\hline 5 & 62 & M & Gastric outlet obstruction & $\begin{array}{l}\text { Metastatic gastric } \\
\text { adenocarcinoma }\end{array}$ & Duodenal stent placement & Success & None \\
\hline \multicolumn{8}{|c|}{$\begin{array}{l}\text { M, male; F, female. } \\
1 \text { Success defined as technically successful stent placement } \\
{ }^{2} \text { Defined as any peri-procedural adverse event, regardless o }\end{array}$} \\
\hline
\end{tabular}

\section{Patient 5: Malignant gastric outlet obstruction}

A 62-year-old male with no significant medical history presented with 2 weeks of abdominal distension, emesis, and melena. Hemoglobin was $6.8 \mathrm{~g} / \mathrm{dL}$ on admission. CT revealed a dilated stomach with significant semisolid-appearing luminal contents, a long segment of marked distal gastric wall thickening, adjacent lymphadenopathy, and peritoneal carcinomatosis. Given the anticipated difficulty with navigating through the retained gastric contents, which did not decrease appreciably with nasogastric tube suction, and our prior successes with the technique, a clear cap was preemptively attached. On endoscopy, a large (7-cm), fungating mass was seen occupying the antrum ( $\triangleright$ Fig.5a). A pinpoint luminal orifice was found and a guidewire successfully passed through it; the cap appeared to improve luminal visualization and thus decrease procedure time. A $23 \mathrm{~mm} \times 90 \mathrm{~mm}$ Evolution (Cook Medical, WinstonSalem, North Carolina, United States) duodenal SEMS was placed across the obstructed segment ( $\mathbf{F i g . 5 b , ~} \mathbf{F i g . 5 c}$, - Fig.5d). Biopsies confirmed invasive adenocarcinoma; the patient opted for palliative chemotherapy and was referred to medical oncology.

\section{Discussion}

Of the five patients in this case series ( $\downarrow$ Table 1 ), three had colonic obstruction and two had gastric outlet obstruction, and all but one case was related to malignant obstruction. In all five cases, use of a clear cap facilitated visualization and successful stent placement in what otherwise may have been technical failure. In addition, in the final case, preemptive use of a clear cap may have decreased the amount of time needed to identify the pinpoint orifice of the obstructed lumen. This can be especially important in cases of colonic obstruction, where prolonged procedure duration (and associated insufflation) may increase risk of perforation, even when $\mathrm{CO}_{2}$ or water is used. To our knowledge, this represents a novel implementation of a clear cap that has not been previously reported in the context of luminal stenting.

\section{Conclusion}

In addition to improving technical success and shortening procedure time, cap-assisted endoscopy as a technical modification also has the benefit of being inexpensive $(\sim 35)$ and safe, particularly when the alternative may be a long and unsuccessful procedure and need for emergent surgical intervention. This simple technical modification may be used as a salvage maneuver when conventional approaches to stenting are unsuccessful, at the outset of cases expected to be particularly challenging, or when alternative emerging techniques such as endoscopic ultrasound-guided gastroenterostomy are contraindicated, not available, or deemed to be less effective or higher risk $[11,12]$. Based on these findings, a prospective study is planned to further investigate the potential benefits (e. g. increased technical success rate and shortened procedure duration) of cap-assisted endoscopic luminal stent placement.

\section{Competing interests}

The authors declare that they have no conflict of interest.

\section{References}

[1] Gaidos JK, Draganov PV. Treatment of malignant gastric outlet obstruction with endoscopically placed self-expandable metal stents. World J Gastroenterol 2009; 15: 4365-4371

[2] Athreya S, Moss ], Urquhart G et al. Colorectal stenting for colonic obstruction: The indications, complications, effectiveness and outcome: 5-year review. Europ J Radiology 2006; 60: 91-94

[3] Ma L, Huang X, Lian J et al. Transparent cap-assisted endoscopic sclerotherapy in esophageal varices: a randomized-controlled trial. Europ J Gastroenterol Hepatol 2018; 30: 626 
[4] Biecker E, Floer M, Heinecke A et al. Novel endocuff-assisted colonoscopy significantly increases the polyp detection rate: a randomized controlled trial. J Clin Gastroenterol 2015; 49: 413

[5] Zhang S, Wang J, Wang J et al. Transparent cap-assisted endoscopic management of foreign bodies in the upper esophagus: A randomized, controlled trial. J Gastroenterol Hepatol 2013; 28: 1339-1342

[6] Trindade AJ, Mella JM, Slattery E et al. Use of a cap in single-balloon enteroscopy-assisted endoscopic retrograde cholangiography. Endoscopy 2015; 47: 453-456

[7] Sumiyama K, Rajan E. Endoscopic caps. Tech Gastrointest Endosc 2006; 8: 28-32

[8] Myung DS, Park CH, Koh HR et al. Cap-assisted ERCP in patients with difficult cannulation due to periampullary diverticulum. Endoscopy 2014; 46: 352-355
[9] Desai M, Sanchez-Yague A, Choudhary A et al. Impact of cap-assisted colonoscopy on detection of proximal colon adenomas: systematic review and meta-analysis. Gastrointest Endosc 2017; 86: 274-281.e3

[10] Tang Z, Zhang DS, Thrift AP et al. Impact of cap-assisted colonoscopy on the learning curve and quality in colonoscopy: a randomized controlled trial. Gastrointest Endosc 2018; 87: 723-732.e3

[11] McCarty TR, Garg R, Thompson CC et al. Efficacy and safety of EUSguided gastroenterostomy for benign and malignant gastric outlet obstruction: a systematic review and meta-analysis. Endosc Int Open 2019; 7: E1474-E14782

[12] Wang G, Liu X, Wang S et al. Endoscopic ultrasound-guided gastroenterostomy: a promising alternative to surgery. J Transl Int Med 2019; 7: 93-99 